\title{
Reverse Köbner response in lichen striatus: the first case described in the literature
}

\author{
Aleksandra Dańczak-Pazdrowska1, Katarzyna Pawlaczyk-Gabriel ${ }^{2}$, Monika Bowszyc-Dmochowska1, \\ Adriana Polańska ${ }^{3}$, Marcin Gabriel ${ }^{4}$, Zofia Gabriel ${ }^{5}$, Ryszard Żaba ${ }^{3}$, Zygmunt Adamski ${ }^{1}$ \\ ${ }^{1}$ Department of Dermatology, Poznan University of Medical Sciences, Poznan, Poland \\ ${ }^{2}$ Department of Hypertension, Angiology and Internal Diseases, Poznan University of Medical Sciences, Poznan, Poland \\ ${ }^{3}$ Department of Dermatology and Venereology, Poznan University of Medical Sciences, Poznan, Poland \\ ${ }^{4}$ Department of General and Vascular Surgery, Poznan University of Medical Sciences, Poznan, Poland \\ ${ }^{5}$ Students Scientific Organization, Poznan University of Medical Sciences, Poznan, Poland
}

Adv Dermatol Allergol 2018; XXXV (4): 434-435

DOI: https://doi.org/10.5114/ada.2018.75840

Lichen striatus is an uncommon, acquired and selflimiting inflammatory dermatosis that presents as small papules, which follows the Blaschko lines. It is considered as a cutaneous mosaicism and the interplay between genetic and environmental factors is postulated. The occasional appearance of lesions after trauma has been reported [1, 2]. Contrary to that, we present a case of lichen striatus in an adult woman in whom eruption disappeared after trauma. We believe it is an example of a rarely reported reverse Köbner response and the first case of this phenomenon in lichen striatus.

A 48-year-old Caucasian female presented with a 6-month history of linear papular eruption of her left lower extremity. The lesion appeared suddenly, initially on the thigh. Over a few weeks it had extended distally to involve the shank and the patient suffered from mild pruritus. On examination there was a linear lesion following Blaschko lines, extending from the proximal thigh to the distal shank which consisted of multiple, flat-topped, erythematous papules of a few millimeters in diameter with a scaly surface (Figure $1 \mathrm{~A}$ ). The other areas of the skin as well as mucous membranes and nails were spared.

The biopsy of the lesional skin from the upper thigh was taken. Histopathology of a biopsy specimen revealed two papules: one composed of spongiotic epidermis with hydropic degeneration of the basal layer and lymphocytic exocytosis with a dense lymphocytic infiltrate in the papillary dermis containing a few melanophages (Figure 2), while the second papule composed of slightly thickened epidermis with interface changes, hyperkeratosis with fo-
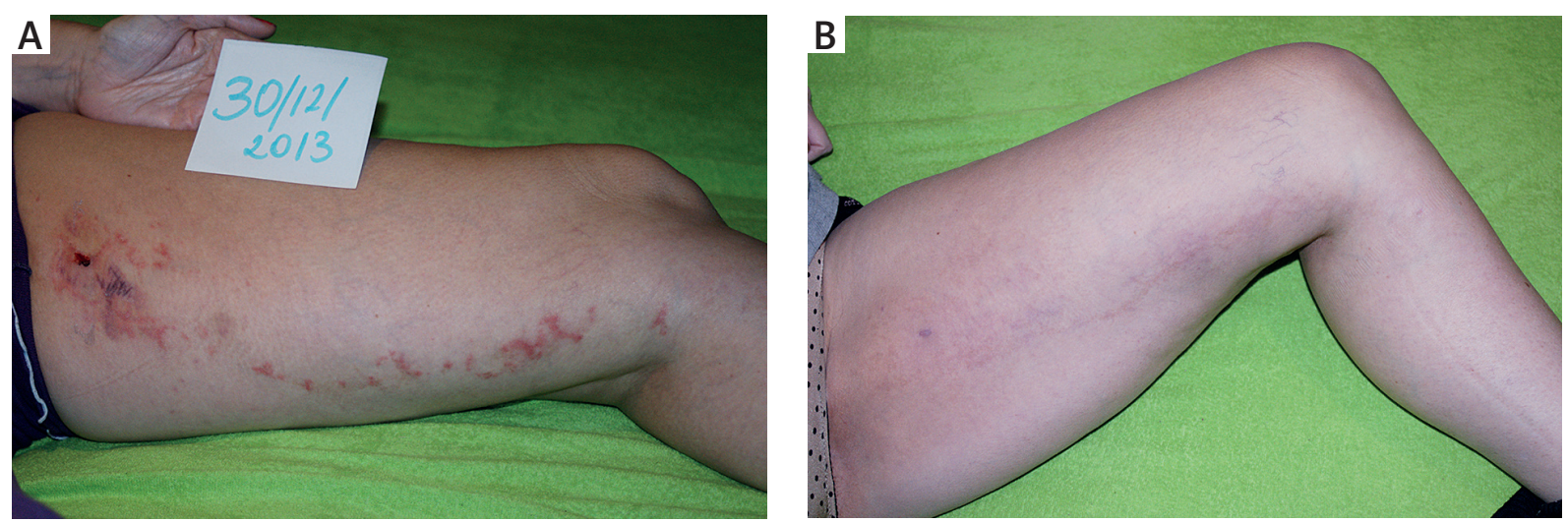

Figure 1. Lichen striatus: A - the beginning clinical state with the visible biopsy area and underneath linear incisions with a scalpel, B - post-inflammatory hyperpigmentation and complete disappearance of lichen striatus 3 months after scalpel incisions

Address for correspondence: Adriana Polańska, Department of Dermatology and Venereology, Poznan University of Medical Sciences, 49 Przybyszewskiego St, 60-355 Poznan, Poland, phone: +48 61869 12 85, e-mail: adriana-polanska@wp.pl Received: 22.05.2017, accepted: 11.06.2017. 
cal parakeratosis and a cluster of necrotic keratinocytes in the upper layers with a mild inflammatory lymphocytic infiltrate underneath.

Based on the clinical presentation and histopathological findings, a diagnosis of lichen striatus was made. The clobetasol propionate $0.05 \%$ ointment was recommended to be applied twice per day. Within 3-4 days after the biopsy the patient noticed disappearance of papules adjacent to the site of the biopsy. At the same time there was no improvement in another part of eruption. As our patient was a doctor (internist) she decided to incise a few next papules with a scalpel. After 2-3 weeks, the lesion cleared with pigmentation and within next 8-12 weeks the pigmentation disappeared completely (Figure 1 B). No recurrence during 3 years' period of follow up was observed.

The exact etiology of lichen striatus remains unknown. It is believed to be a manifestation of cutaneous mosaicism. It is postulated that the clone of aberrant keratinocytes is attacked after contact with the triggering factor (mostly after viral infections) which affects previous tolerance [1]. The immunosuppressive effect of UV radiation was also suggested by Ciconte [3]. However cases presented by these authors may have been another example of trauma-induced lichen striatus as trauma has been reported as the triggering factor in lichen striatus as well $[1,2]$.

In our case the lesion disappeared after repeated trauma. Contrary to Köbner phenomenon in which new eruptions typical of an existing dermatosis are observed at sites of injuries, such disappearance of the lesions after trauma is called reverse Köbner response [4]. For the first time it was described in psoriatic patients [5]. According to Eyre et al., as many as $67 \%$ of patients suffering from plaque psoriasis undergoing trauma presented resolution of skin lesions. Those patients in whom reverse Köbner response was observed had more stable disease and lower Psoriasis Area and Severity Index (PASI) than those with Kőbner phenomenon [6]. Casuistic reports on reverse Köbner phenomenon in vitiligo [7, 8] and leukocytoclastic vasculitis were also published [9]. The skin biopsy provoked reverse Köbner response in an interstitial variant of granuloma annulare [10].

\section{Conflict of interest}

The authors declare no conflict of interest.

\section{References}

1. Shepherd V, Lun K, Strutton G. Lichen striatus in an adult following trauma. Australian J Dermatol 2005; 46: 25-8.

2. Coto-Segura P, Costa-Romero M, Gonzalvo P, et al. Lichen striatus in an adult following trauma with central nail plate involvement and its dermoscopy features. Int I Dermatol 2008; 47: 1324-5.

3. Ciconte A, Bekhor P. Lichen striatus following solarium exposure. Australian J Dermatol 2007; 48: 99-101.

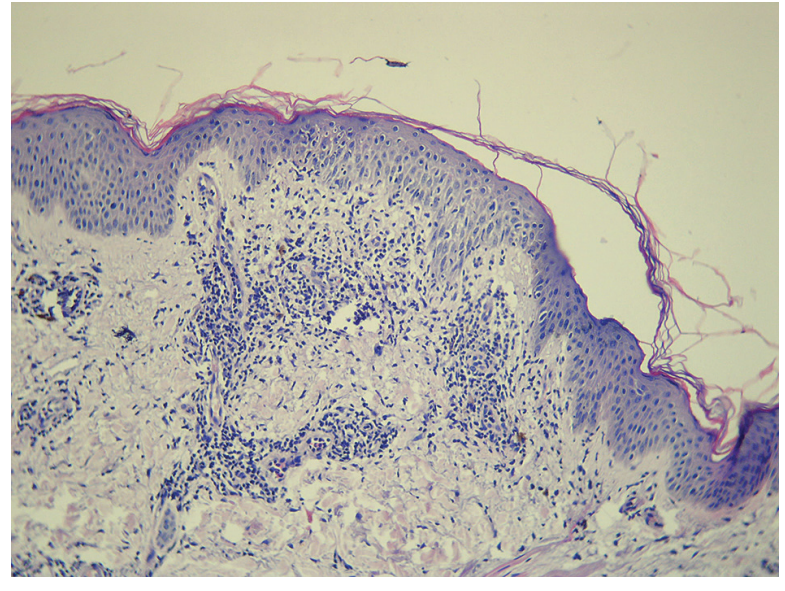

Figure 2. Spongiotic epidermis with hydropic degeneration of the basal layer and lymphocytic exocytosis with a dense lymphocytic infiltrate in the papillary dermis containing a few melanophages $(H+E$, magnification 200x)

4. Kannangara AP, Yosipovitch G, Fleischer Jr AB. Proposed classification for Koebner, wolf isotopic, renbok, Koebner nonreaction, isotopic nonreaction and other related phenomen. Dermatol Online J 2014; 20: pii: 13030/qt96s656b4.

5. Camargo CM, Brotas AM, Ramos-e-Silva M, et al. Isomorphic phenomenon of Koebner: facts and controversies. Clin Dermatol 2013; 31: 741-9.

6. Eyre RW, Krueger GG. Response to injury of skin involved and uninvolved with psoriasis, and its relation to disease activity: Koebner and 'reverse' Koebner reactions. Br J Dermatol 1982; 106: 153-9.

7. Malakar S, Dhar S. Spontaneous repigmentation of vitiligo patches distant from the autologous skin graft sites: a remote reverse Koebner's phenomenon? Dermatology 1998; 197: 274.

8. Laxmisha C, Kumari R, Thappa DM. Satellite repigmentation (remote reverse Koebner phenomenon). Dermatol Surg 2006; 32: 596.

9. Yadav S, De D, Kanwar AJ. Reverse koebner phenomenon in leukocytoclastic vasculitis. Indian J Dermatol 2011; 56: 598-9.

10. Naveen KN, Varadraj VP, Sharatchandra BA, et al. Remote reverse Koebner phenomenon in generalized granuloma annulare. Indian Dermatol Online J 2014; 5: 219-21. 